Chirurg 2021 $\cdot 92: 478$

https://doi.org/10.1007/s00104-021-01402-z

Angenommen: 15. März 2021

Online publiziert: 19. April 2021

(c) Springer Medizin Verlag GmbH, ein Teil von Springer Nature 2021

\section{Originalpublikation}

Yamada S, Fujii T, Sonohara F et al (2021) Safety of combined division vs separate division of the splenic vein in Patients undergoing distal Pancreatectomy: a noninferiority randomized clinical trial. JAMA Surg. https://doi.org/10. 1001/jamasurg.2021.0108

Hintergrund. Bei der Pankreaslinksresektion werden das Pankreasparenchym und die Milzvene in der Regel separat voneinander durchtrennt. Nach der Etablierung der Staplerresektion des Pankreas werden an einigen Kliniken die Milzvenen- und die Pankreasdurchtrennung gemeinsam mittels eines Staplers durchgeführt. $\mathrm{Ob}$ durch diese Kombination postoperative Nachblutungen basierend auf Pankreasfisteln vermehrt auftreten, ist hierbei bisher ungeklärt.

Methoden.Die COSMOS-DP(Combined Resection vs Separated Resection After Mobilization of the Splenic Vein During Distal Pancreatectomy)-Studie wurde als eine multizentrische, randomisierte Phase-3-Nichtunterlegenheitsstudie an 45 japanischen Zentren durchgeführt. Der primäre Endpunkt war das Auftreten von Grad-B- und -C-Pankreasfisteln. Sekundäre Endpunkte waren intraoperative Kennzahlen wie Operationszeit, Blutverlust, Blutstillung und Integrität der Klammernaht und postoperative Kennzahlen wie Drainagedauer, Krankenhausverweildauer, Komplikationen mit intraabdominalen Nachblutungen und Mortalität. Komplikationen wurden 6 Monate postoperative nach den ISGPS-Klassifikationen und der ClavienDindo-Klassifikation erfasst. Es wurden

Thomas Schmidt • Markus W. Büchler

Klinik für Allgemeine, Viszerale und Transplantationschirurgie, Universität Heidelberg, Heidelberg, Deutschland

\title{
Sichere kombinierte Pankreas- und Milzvenenresektion bei der distalen Pankreasresektion
}

Patienten mit Karzinomen, IPMNs, neuroendokrinen Tumoren, muzinös zystischen Neoplasien und Metastasen des Pankreasschwanzes eingeschlossen, die sowohl offen als auch laparoskopisch operiert wurden.

Ergebnisse. Es wurden 318 Patienten in die Studie eingeschlossen, hiervon 159 Patienten in Arm A mit einer separaten Durchtrennung und 159 in Arm B mit einer gemeinsamen Durchtrennung von Pankreas und Milzvene. Die Patientencharakteristika inklusive der Dicke des Pankreas und des Durchmessers des Pankreasganges unterschieden sich nicht zwischen den Gruppen. Es gab keine Unterschiede im operativen Vorgehen und bei operativen Parametern inklusive der Operationszeit oder der Beschaffenheit des Pankreas. Es zeigten sich keine Unterschiede bei dem primären Endpunkt, den Grad-B/C-Pankreasfisteln. Sie traten in 27,1 \% bzw. 28,6\% der Fälle auf, wodurch eine Nichtunterlegenheit der gemeinsamen Durchtrennung gezeigt wurde. In der Kombinationsgruppe wurde häufiger eine Blutstillung an der Klammernaht durchgeführt und es kam häufiger zu Verletzungen des Pankreas, beides ohne statistische Signifikanz. Postoperative Nachblutungen waren mit $1,3 \%$ in beiden Gruppen gleich selten. Es kam zu keinen Nachblutungen aus der Milzvene in beiden Gruppen.

\section{Kommentar}

Die Autoren folgern, dass eine kombinierte Milzvenen- und Pankreasresekti- on mit dem Stapler sicher durchgeführt werden kann.

Die Rate der postoperativen Nachblutungen liegt mit 1,3\% auf einem niedrigen Niveau im internationalen Vergleich. Allerdings bleibt festzuhalten, dass auch kein Vorteil für eine kombinierte Resektion gefunden wurde, insbesondere keine Reduktion der Operationszeit oder des intraoperativen Blutverlustes. Weiter bleibt kritisch anzumerken, dass sich diese Operationstechnik nur für Tumoren eignet, die sich deutlich linksseitig des venösen Konfluenz befinden, da es sonst zu einer Einengung durch eine kombinierte Resektion kommen kann. In der Studie wurde die Resektionslinie mindestens $1 \mathrm{~cm}$ linksseitig der Pfortader gewählt. Obwohl die Anzahl der Grad-B/C-Pankreasfisteln höher war, als die Autoren initial angenommen hatten, liegt diese Anzahl in dem bekannten Bereich innerhalb randomisierter klinischer Studien. Dies bestätigt einmal mehr die Notwendigkeit weiterer Optimierung der Operationstechnik.

\section{Korrespondenzadresse}

Prof. Dr. Dr. med. Thomas Schmidt

Klinik für Allgemeine, Viszerale und

Transplantationschirurgie, Universität Heidelberg

Im Neuenheimer Feld 420, 69120 Heidelberg, Deutschland

Thomas.Schmidt1@med.uni-heidelberg.de

Interessenkonflikt. T. Schmidt und M.W. Büchler geben an, dass kein Interessenkonflikt besteht. 